\title{
Optical analysis for few TMDC materials
}

\author{
MEHUL DAVE \\ Natubhai V. Patel College of Pure and Applied Sciences, Vallabh Vidyanagar 388 120, India
}

MS received 9 September 2014; accepted 5 February 2015

\begin{abstract}
The transition metal dichalcogenides possess layered structure of Se-M-Se $(\mathrm{M}=\mathrm{Nb}, \mathrm{Mo}$, Ta and W) sandwich interact with each other by van der Waal forces and can also provide sites for intercalation. Because of their technological importance, lubricants, catalysts, battery cathodes and electrodes in the photoelectrochemical solar cells, much attention has gone in the studies of growth of these materials in crystalline and nanocrystalline forms. In the present work we report the growth of $\mathrm{NbSe}_{2}, \mathrm{MoSe}_{2}, \mathrm{TaSe}_{2}$ and $\mathrm{WSe}_{2}$ single crystals and determine the optical bandgap using optical absorption. The optical absorption of as-grown crystals has been measured at room temperature near the fundamental absorption edge. Both direct and indirect transitions are involved in the absorption process. The indirect transition was found to be allowed with two phonons involved in the process. The direct and indirect energy gaps and phonon energies for all crystals have been estimated. The results obtained are discussed in detail.
\end{abstract}

Keywords. Transition metal diachalcogenides; optical parameters; bandgap.

\section{Introduction}

To understand the nature of semiconductors one must consider what happens when similar atoms are brought together to form a solid, such as a crystal. As two similar atoms approach each other, the wave function of their electrons begins to overlap. The energy distribution of the states depends strongly on the interatomic distance. It is the extent of the energy gap and the relative availability of electrons that determine whether a solid is a metal, a semiconductor or an insulator. In a semiconductor the energy gap usually extends over less than about $3 \mathrm{eV}$ and the density of electrons in the upper band is usually less than $10^{20} \mathrm{~cm}^{-3}$.

As the interatomic distance in a crystal is not isotropic but rather varies with the crystallographic direction, one would expect this directional variation to affect the banding of states. Thus although the energy gap, which characterizes a semiconductor, has the same minimum value in each unit cell, its topography within each unit cell can be extremely complex. In compound semiconductors a deviation from stoichiometry generates donors or acceptors depending on whether it is the cation or anion, which is in excess. However, it has been shown that it is not the excess ion but rather the vacancy, which determines whether the material is n-type or p-type in nature. ${ }^{1}$ The band structures of several real and dummy layered transition metal dichalcogenides (TMDCs), $\mathrm{NbSe}_{2}, \mathrm{TaS}_{2}$ and $\mathrm{TaSe}_{2}$, were calculated by using the tightbinding method within the extended Hückel approximation (EHT). ${ }^{2}$ Angle-resolved photoemission at $21.2 \mathrm{eV}(\mathrm{HeI})$ and $16.8 \mathrm{eV}(\mathrm{NeI})$ is used to map the band structure of $2 \mathrm{H}-$ $\mathrm{NbSe}_{2}$, and the results are compared with various theoretical

(addave1977@yahoo.com) calculations. ${ }^{3}$ At $33 \mathrm{~K} 2 \mathrm{H}-\mathrm{NbSe}_{2}$ distorts into an incommensurate charge density wave (CDW) state. The Raman spectrum shows amplitude modes of $\mathrm{A}$ and $\mathrm{E}$ symmetry near $40 \mathrm{~cm}^{-1}$ induced by the CDW. ${ }^{4}$ The optical absorption of $\mathrm{NbSe}_{2}$ was measured by a scanning calorimetric technique in the spectral range of 0.38 to $3.2 \mathrm{eV} .^{5}$ Angle-resolved photoemission model for the layered TMDCs has been proposed, which accounts for many of the observed properties of these materials. ${ }^{6}$ On the basis of this band scheme, the group V TMDC, $2 \mathrm{H}-\mathrm{NbSe}_{2}$, is expected to display metallic properties arising from a narrow half-filled $d_{z^{2}}$ band. This simple band picture has been further refined by photoemission measurement ${ }^{7-9}$ and by a detailed study of the development of the $\mathrm{Nb}$-based $4 \mathrm{~d}$ bands, starting with a $\mathrm{NbSe}_{2}$ cluster. ${ }^{10}$ Angle-resolved inverse photoemission spectra have been measured at room temperature on the layered TMDCs, $2 \mathrm{H}-\mathrm{NbSe}_{2}$ and $2 \mathrm{H}-\mathrm{TaSe}_{2}$ in the isochromat mode. ${ }^{11}$ Layered TMDCs of the type $\mathrm{MX}_{2}(\mathrm{M}=$ group-IV, $-\mathrm{V},-\mathrm{VI}$ atoms and $\mathrm{X}=$ chalcogen atoms) exhibit interesting physical and structural properties as have been reviewed by many authors. ${ }^{12}$ For example, a CDW was reported on $\mathrm{TaSe}_{2}{ }^{13}$ and later on other metallic TMDC. The optical bandgap of a semiconducting material play an important role in determining the photo conversion efficiency of a solar cell fabricated with it. Optical bandgap (direct as well as indirect) can be determined by the following methods.

(1) Optical absorption

(2) Photo electrochemical methods

(a) Quantum yield $\eta v s$. $h v$ plot

(b) Action spectrum

(c) Capacitance measurements 
(3) Intrinsic conduction measurement at high temperature

(4) Photoemission and

(5) Band structure calculations.

However, looking at the importance of optical bandgap in these materials, we have carried out a detailed study on the determination of optical bandgap in these materials by optical absorption. The results thus obtained have been described and discussed in this article.

\section{Experimental}

The layered single crystals of TMDCs $\mathrm{MX}_{2}\left(\mathrm{M}=\mathrm{IV}_{\mathrm{b}} \mathrm{V}_{\mathrm{b}}\right.$ transition metal, $\mathrm{X}=\mathrm{S}$, Se and Te) have been grown by the chemical vapour transport (CVT) technique using iodine as a transporting agent. The stoichiometry of as-grown crystals has been confirmed on the basis of energy-dispersive analysis of X-ray. X-ray diffraction study has been performed for the structural characterization of as-grown crystals. The X-ray diffractogram was obtained with Philips X-ray diffractometer (model: PW1820) employing $\mathrm{CuK}_{\alpha}$ radiation. All samples were grown by CVT technique. As-grown samples exhibit p-type conductivity with a whole concentration of $10^{13}$ to $10^{16} \mathrm{~cm}^{-3}$ at room temperature.

Thin samples for absorption measurement were obtained by cleavage. The $a$ and $b$ axes were contained in the plane of cleavage. The optical absorption data were obtained by means of UV-VIS-NIR Spectrophotometer (Make: Perkin Elmer, model: Lambda-19). Prior to measurement, samples were oriented both optically and by back reflection Laue techniques. Measurements were performed at room temperature with the electric field of the incident light parallel to $a$ and $b$ crystallographic axes, respectively. Measurement along the $c$-axis was not performed because the crystal structure did not permit cutting and polishing. The energy range of incident photons was extended from 1.0 to $1.5 \mathrm{eV}$.

For the relationship that exists for possible transitions across the energy gap of semiconductor, the absorption coefficient ' $\alpha$ ' is proportional as follows, ${ }^{14}$

$$
\alpha h v=A\left(h v-E_{\mathrm{g}}\right)^{r}
$$

for direct transitions and

$$
\alpha h v=\sum_{j} B_{j}\left(h v-E_{\mathrm{g}}^{\prime} \pm E_{\mathrm{pj}}\right)^{r}
$$

for indirect transitions.

Here ' $\alpha$ ' is the absorption coefficient, h $v$ the energy of the incident photon, $E_{\mathrm{g}}$ the energy for the direct transition and $E_{\mathrm{g}}^{\prime}$ the energy for indirect transition and $E_{\mathrm{pj}}$ the energies of the phonons assisting at indirect transition. A and $\mathrm{B}$ are parameters depending in a more complicated way on temperature, photon energy and phonon energies $E_{\mathrm{p}}$.

However, for the analysis of the experimental results obtained at constant temperature, equations (1) and (2) are sufficient and they are most often used while interpreting results on absorption spectra obtained from semiconducting materials. The exponent ' $r$ ' in the above equations depends upon whether the transition is symmetry allowed or not and the constants A and B will assume different values for the allowed and forbidden transitions.

For indirect transitions the detailed form of equation $(2)^{14}$ is given as,

$$
\begin{aligned}
\alpha_{i}= & \sum_{i=1}^{2}\left\{\frac{B_{\mathrm{a} i}}{E}\left(\frac{1}{e^{\theta_{i} / T}-1}\right)\left(E-E_{\mathrm{g}}^{\prime}+k \theta_{i}\right)^{r}\right. \\
& \left.+\frac{B_{\mathrm{e} i}}{E}\left(\frac{1}{1-e^{\theta_{i} / T}}\right)\left(E-E_{\mathrm{g}}^{\prime}-k \theta_{i}\right)^{r}\right\}
\end{aligned}
$$

where $B_{\mathrm{a} i}$ and $B_{\mathrm{e} i}$ are coefficients associated with absorption and emission of $i$ th phonon, $E$ the photon energy, $E_{\mathrm{g}}^{\prime}$ the indirect energy gap and $\theta_{i}$ is a phonon equivalent temperature defined by the equation,

$$
\theta_{i}=\frac{E_{\mathrm{p} i}}{k}
$$

where $E_{\mathrm{p} i}$ being the $i$ th phonon energy.

In these cases the density of states is a constant independent of the energy, and the expressions showing the dependence of $\alpha$ in terms of direct and indirect transitions get $\operatorname{modified}^{7}$ as

$$
\alpha=A^{\prime}\left(h v-E_{\mathrm{g}}\right)^{r}
$$

for direct transition and

$$
\begin{aligned}
\alpha_{i}= & \sum_{i=1}^{2}\left\{B_{\mathrm{a} i}^{\prime}\left(\frac{1}{e^{\theta_{i} / T}-1}\right)\left(E-E_{\mathrm{g}}^{\prime}+k \theta_{i}\right)^{r}\right. \\
& \left.+B_{\mathrm{e} i}^{\prime}\left(\frac{1}{1-e^{\theta_{i} / T}}\right)\left(E-E_{\mathrm{g}}^{\prime}-k \theta_{i}\right)^{r}\right\}
\end{aligned}
$$

for indirect transition.

\section{Band tailing}

While impurity-band formation is an obvious consequence of increased impurity concentration, another important effect occurs that is a perturbation of the bands by the formation of tails of states extending the bands into the energy gap. The problem of band tailing has received much theoretical attention. ${ }^{15}$ An ionized donor exerts an attractive force on the conduction electrons and a repulsive force on the valance holes. As impurities are distributed randomly in the host crystal, the local interaction will be more or less strong depending on the local crowding of impurities. Dislocations are also usually present in crystals. They occur at the edge of an extra plane of atoms. The misfit of such an extra plane results in compressional and dilational strains, with the consequent onset of both lowering and raising of the potentials in the neighbourhood of the dislocation. Hence, one can say that impurities will induce tails in the density 
states by perturbing the band edge via deformation potential, via coulomb interaction, and by forming a band of impurity states. The momentum conserving transition between parabolic bands results in absorption edge, which obeys equation (1) ( $r=1 / 2)$, i.e., where the absorption constant increases with the square root of the photon energy in excess of the gap energy. For direct transition one expects no absorption below the energy gap and, therefore, a steeply rising absorption edge. However, in practice one usually finds an exponentially increasing absorption edge. ${ }^{16}$

In a number of materials, it is found that $\mathrm{d}(\ln \alpha) / \mathrm{d}(h v)=$ $1 / \mathrm{kT}$; this is known as Urbach's rule. ${ }^{17}$ In the case of degenerate p-type material, the Fermi level is taken to be in the parabolic portion of the valence band, so that the perturbed part of the valence band lies above the Fermi level. Then the density of initial states, $N_{\mathrm{i}}$ is proportional to $\left|E_{\mathrm{v}}\right|^{1 / 2}$, where $E_{\mathrm{v}}$ is the energy of the state with respect to what would be the edge of the parabolic valence band as shown in figure 1.

\section{Results and discussion}

The absorption spectra of $\mathrm{MSe}_{2}(\mathrm{M}=\mathrm{Nb}, \mathrm{Mo}$, Ta and $\mathrm{W})$ single crystal are shown in figure 2 . A careful study of these

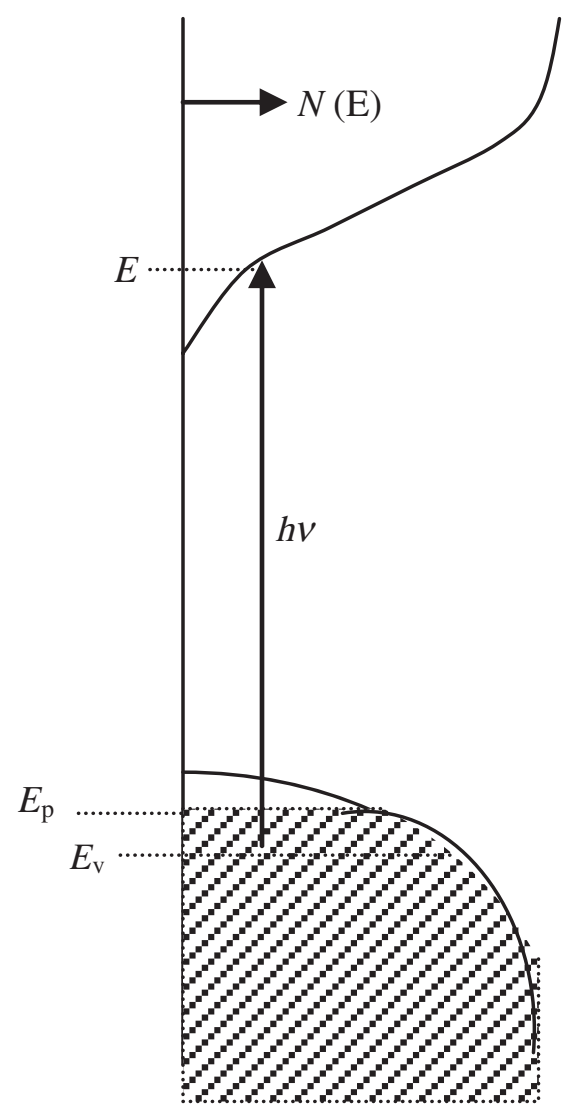

Figure 1. Energy diagram illustrating how absorption probes the conduction band tail of states in a p-type semiconductor. The tail of the valence band has been absorption process. spectra reveals the presence of absorption edges in the spectral range 700 to $850 \mathrm{~nm}$. In order to analyse the results from this spectra in the vicinity of the absorption edge on the basis of two- as well as three-dimensional model, values of
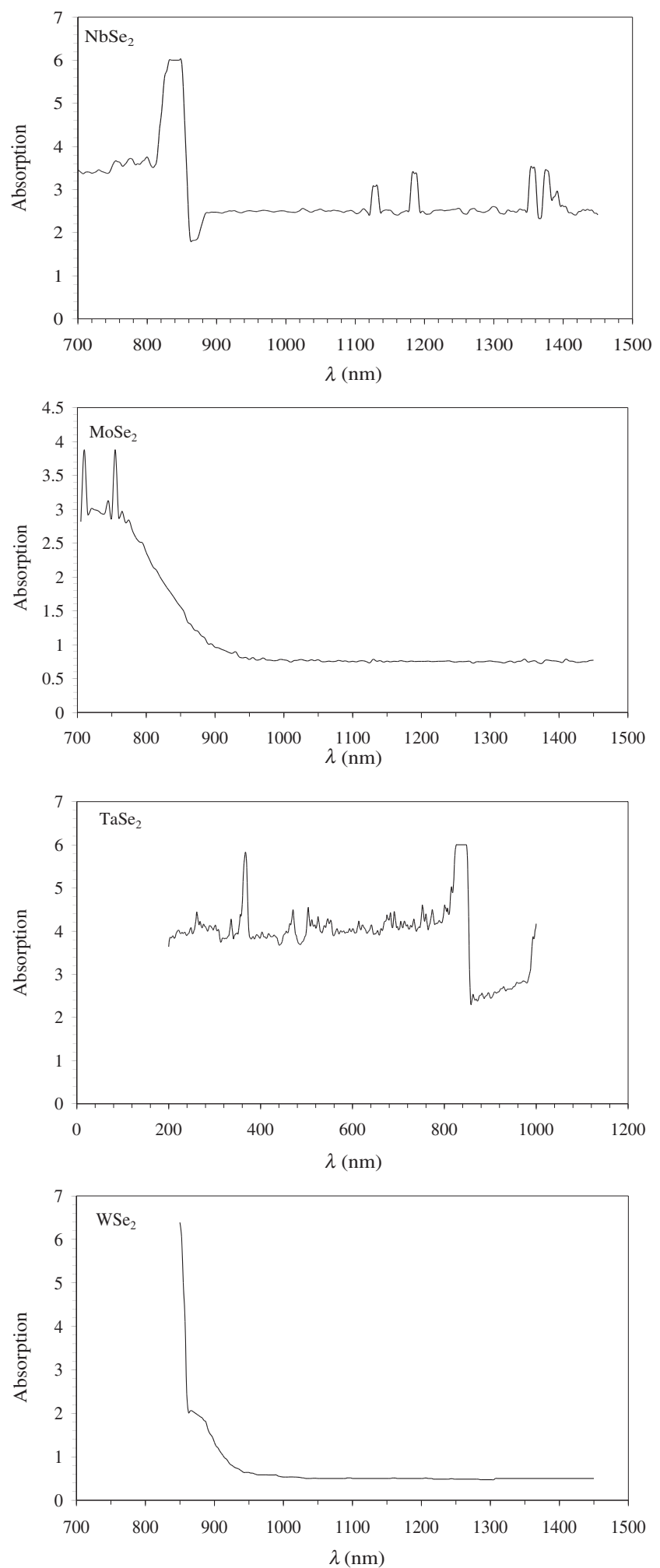

Figure 2. The graph of optical spectrum for as-grown crystals of $\mathrm{MSe}_{2}(\mathrm{M}=\mathrm{Nb}$, Mo, Ta and $\mathrm{W})$. 

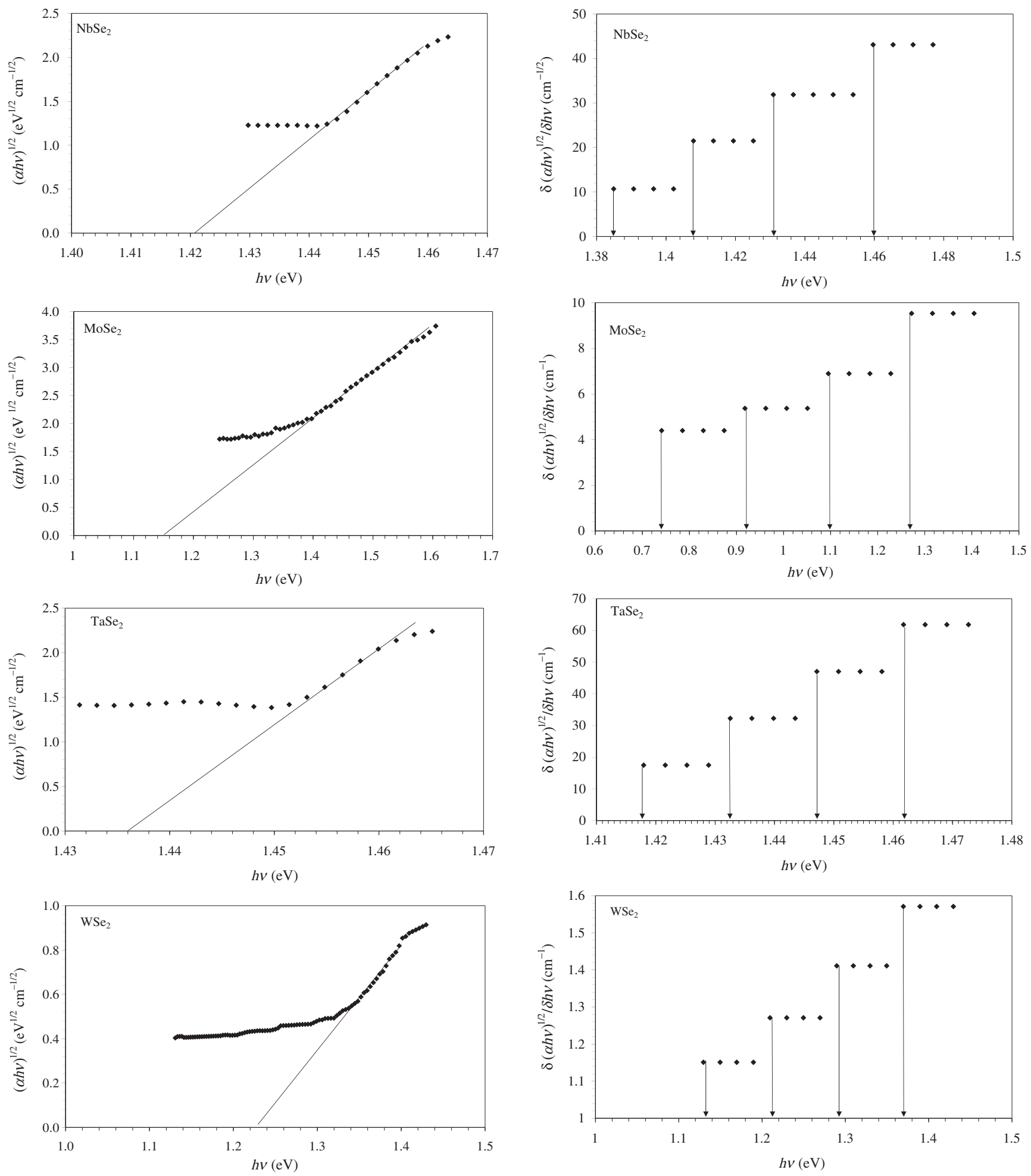

Figure 3. The graph of $(\alpha h v)^{1 / 2} v s$. $h v$ for as-grown crystals of $\mathrm{MSe}_{2}$.

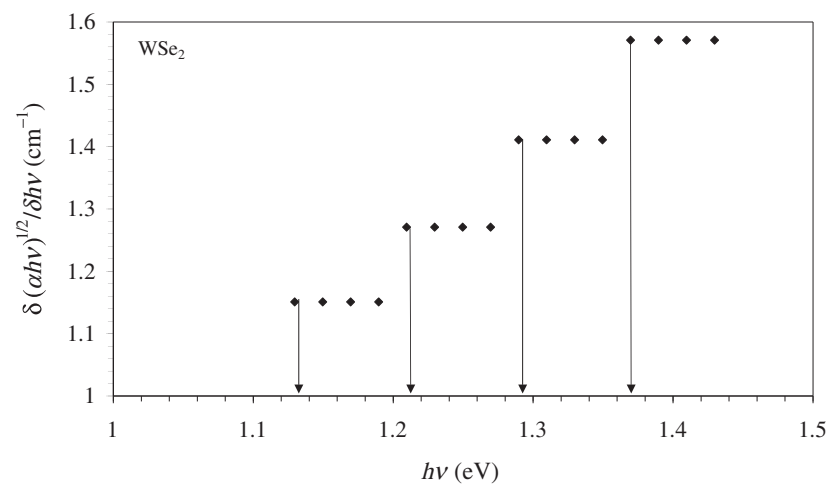

Figure 4. The spectral variation of $\delta(\alpha h v)^{1 / 2} / \delta h v v s$. $h v$ for as-grown crystals of $\mathrm{MSe}_{2}(\mathrm{M}=\mathrm{Nb}, \mathrm{Mo}$, Ta and $\mathrm{W})$.

absorption coefficient $\alpha$ were determined at every interval of $5 \mathrm{~nm}$. The interpretation of the experimental results viz. the dependence of absorption coefficient ' $\alpha$ ' in terms of direct and indirect transitions can be performed with the help of

formulae 1 and 2 using the values of $r$ equal to $1 / 2$ and 2, respectively.

The $\mathrm{MSe}_{2}(\mathrm{M}=\mathrm{Nb}, \mathrm{Mo}, \mathrm{Ta}$ and $\mathrm{W})$ single crystal shows the spectral variation of $(\alpha h v)^{1 / 2} v s$. $h v$, as shown in figure 3 . 
Table 1. Optical parameters for as-grown crystals of $\mathrm{MSe}_{2}$ $(\mathrm{M}=\mathrm{Nb}, \mathrm{Mo}$, Ta and $\mathrm{W})$.

\begin{tabular}{lcccc}
\hline Parameters & $\mathrm{NbSe}_{2}$ & $\mathrm{MoSe}_{2}$ & $\mathrm{TaSe}_{2}$ & $\mathrm{WSe}_{2}$ \\
\hline$E_{1}(\mathrm{eV})$ & 1.38 & 0.74 & 1.42 & 1.13 \\
$E_{2}(\mathrm{eV})$ & 1.41 & 0.92 & 1.43 & 1.21 \\
$E_{3}(\mathrm{eV})$ & 1.43 & 1.10 & 1.45 & 1.29 \\
$E_{4}(\mathrm{eV})$ & 1.46 & 1.27 & 1.46 & 1.37 \\
$E_{\mathrm{p} 1}(\mathrm{eV})$ & 0.04 & 0.27 & 0.02 & 0.12 \\
$E_{\mathrm{p} 2}(\mathrm{eV})$ & 0.01 & 0.09 & 0.01 & 0.04 \\
$\theta_{1}(\mathrm{~K})$ & 464 & 3134 & 232 & 1393 \\
$\theta_{2}(\mathrm{~K})$ & 116 & 1044 & 116 & 464 \\
$E_{\mathrm{g}}(\mathrm{eV})($ direct $)$ & 1.44 & 1.40 & 1.45 & 1.41 \\
$E_{\mathrm{g}}^{\prime}(\mathrm{C})(\mathrm{eV})$ & 1.42 & 1.00 & 1.44 & 1.23 \\
$E_{\mathrm{g}}^{\prime}(\mathrm{E})(\mathrm{eV})$ & 1.42 & 1.14 & 1.43 & 1.23 \\
Band tailing, $E_{0}$ & 0.03 & 0.04 & 0.03 & 0.03 \\
Actual bandgap $\left(E_{\mathrm{g}}^{\prime}-E_{0}\right)(\mathrm{eV})$ & 1.39 & 1.10 & 1.40 & 1.20 \\
\hline
\end{tabular}

$E_{\mathrm{g}}^{\prime}(\mathrm{C})=$ indirect bandgap from calculation; $E_{\mathrm{g}}^{\prime}(\mathrm{E})=$ indirect bandgap from extrapolation; $\theta_{1}$ and $\theta_{2}$ can be determined by using equation (4).

As the curves indicate discontinuous straight lines, it is quite possible that they represent indirect interband transitions involving the emission and absorption of phonons.

Accordingly, from the graphical differentiation of the data presented in figure 3 for $\mathrm{MSe}_{2}(\mathrm{M}=\mathrm{Nb}, \mathrm{Mo}$, Ta and $\mathrm{W})$ single crystal, the dependence of the derivatives $\delta(\alpha h v)^{1 / 2} / \delta h v$ on $h v$ has been shown in figure 4. It can be clearly seen from this figure that the derivatives are step function of energy with four steps well defined in the range:

$$
\begin{aligned}
& E_{1}<E<E_{2}, \quad E_{2}<E<E_{3}, \\
& E_{3}<E<E_{4} \text { and } E_{4}<E .
\end{aligned}
$$

These values of $E_{1}, E_{2}, E_{3}$ and $E_{4}$ indicate the points of discontinuities in the plots of $\delta(\alpha h v)^{1 / 2} / \delta h v$ vs. $h v$.

The indirect energy gaps obtained from these values of $E_{1}$, $E_{2}, E_{3}$ and $E_{4}$ are given by

$$
\begin{aligned}
& E_{\mathrm{g}}^{\prime}=\frac{E_{1}+E_{4}}{2}=\frac{E_{2}+E_{3}}{2}, \\
& E_{\mathrm{p} 1}=\frac{E_{4}-E_{1}}{2} \text { and } E_{\mathrm{p} 2}=\frac{E_{3}-E_{2}}{2} .
\end{aligned}
$$

The values of indirect bandgap $E_{\mathrm{g}}^{\prime}$ and phonon energies thus obtained are presented in table 1 . The values of the $E_{\mathrm{g}}^{\prime}$ can also be obtained from the intersection of the linear portion of the curves with energy axis for zero absorption as shown in figure 4 . These values closely match with the values obtained from equation (7).
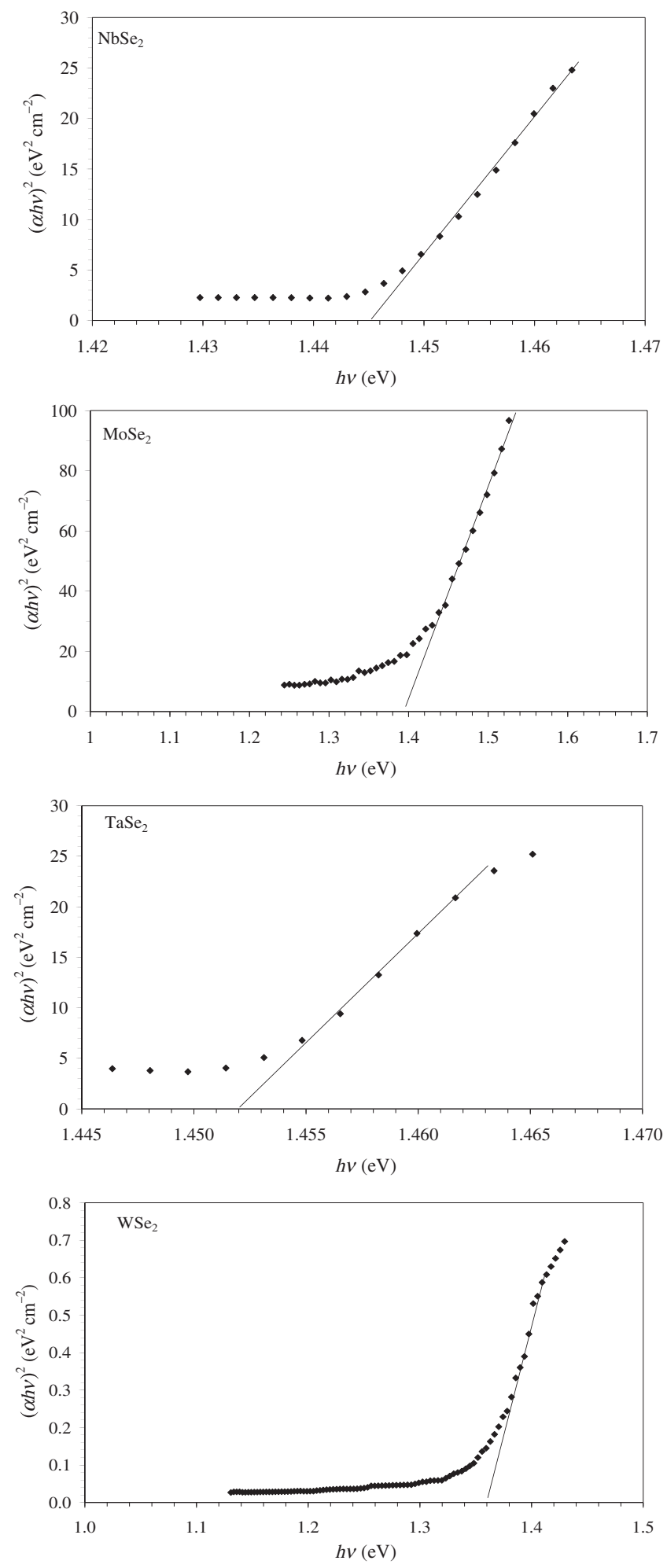

Figure 5. The graph of $(\alpha h v)^{2} v s$. $h v$ for as-grown crystals of $\mathrm{MSe}_{2}$.

For the determination of the direct bandgap $E_{\mathrm{g}}$, the spectral variation of $(\alpha h v)^{2} v s$. $h v$, as shown in figure 5, was studied. The values of direct bandgap $E_{\mathrm{g}}$ obtained from the intersection of the linear portion of the curves in figure 6 are shown in table 1 . 

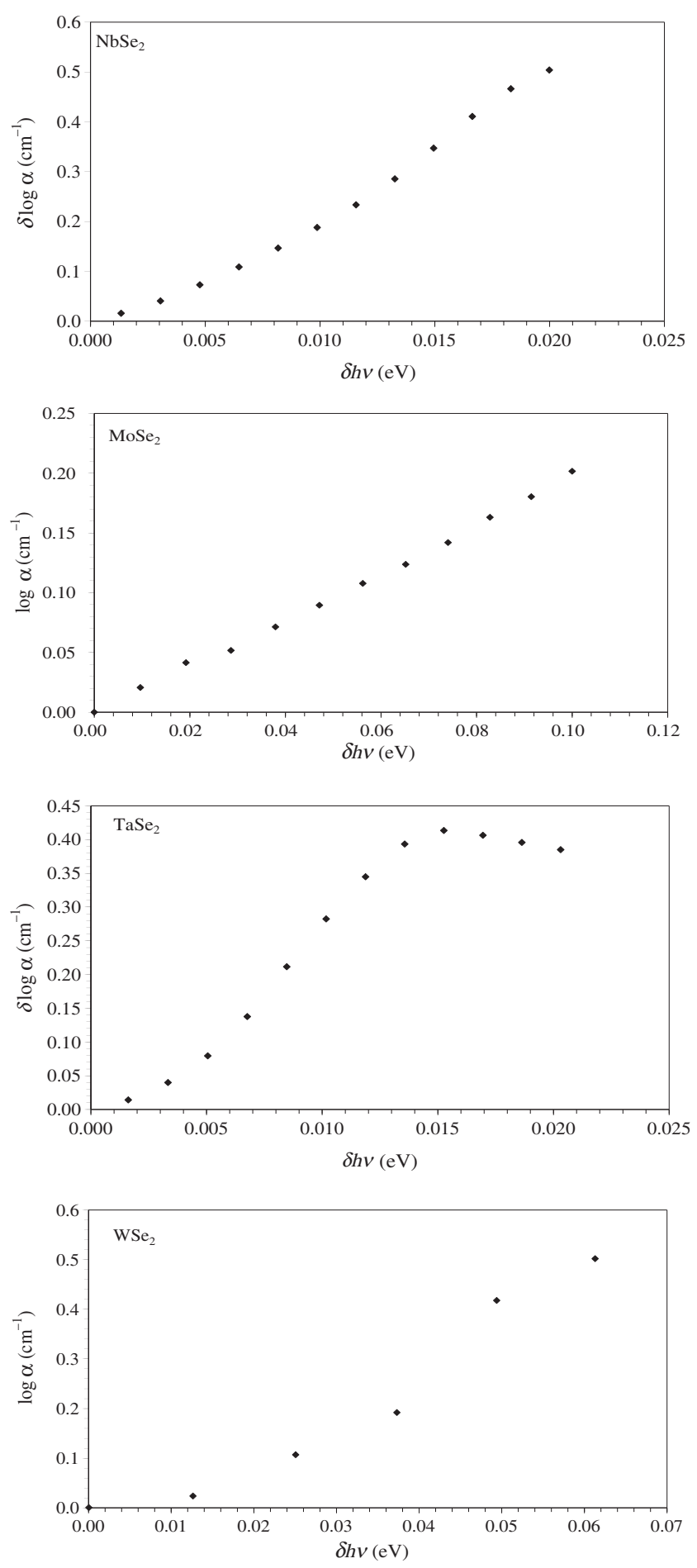

Figure 6. The graph of $\delta \log \alpha v s$. $\delta h v$ for as-grown crystals of $\mathrm{MSe}_{2}(\mathrm{M}=\mathrm{Nb}, \mathrm{Mo}$, Ta and $\mathrm{W})$.

\section{Conclusion}

These both direct as well as we observed that both direct as well as indirect symmetries allowed transitions and gave a good account of the optical absorption edge in $\mathrm{MSe}_{2}(\mathrm{M}=$ $\mathrm{Nb}, \mathrm{Mo}, \mathrm{Ta}$ and $\mathrm{W}$ ) single crystals. In this context, we have the following conclusions:

1. The analysis of the optical absorption in these crystals near the fundamental edge has been shown that both direct as well as indirect transitions takes place in these compounds.

2. The indirect transitions are involving two different phonons.

3. The actual values of the bandgap have been determined with the help of band tailing.

\section{References}

1. Parada N J and Pratt G W Jr 1969 Phys. Rev. Lett. 22180

2. Subba Rao G V and Shafer M V 1979 Physics and chemistry of materials with layered structures Lavy F (ed) (Holland: Reidel D. Dordrecht) 699

3. Jakovidis G, Riley J D and Leckey R C G 1992 J. Electron Spectrosc. Relat. Phenom. 58119

4. Sooryakumar R and Klein M V 1981 Physica $B+C \mathbf{1 0 5}$ 422

5. Bachmann R, Kirsch H C and Geballe T H 1971 Solid State Commun. 957

6. Wilson J A and Yoffe A D 1969 Adv. Phys. 18193

7. McMenamin J C and Spicer W E 1972 Phys. Rev. Lett. 29 1501

8. Williams R H 1973 J. Phys. C 6 L32

9. Weitheim G K, Di Salvo F J and Buchanan D N E 1973 Solid State Commun. 131225

10. Mattheiss L F 1973 Phys. Rev. B8 3719

11. Shu-Hui Cai and Chun-Wan Liu 1996 J. Mol. Struct. Theochem. $\mathbf{3 6 2} 379$

12. Smith N V and Traum M M 1975 Phys. Rev. B11 2087

13. Wilson J A, Dolvo F J and Mahahan S 1974 Phys. Rev. Lett. 32 882

14. Pankove J I 1975 Optical processes in semiconductors (New York: Dover Publ. Inc.) p 1

15. Halperin B I and Lax M 1966 Phys. Rev. 148772

16. Pankove J I 1965 Phys. Rev. 140A 2059

17. Urbach F 1953 Phys. Rev. 921324 